\title{
ON RUNGE-KUTTA-NYSTROM FORMULAE WITH CONTRACTIVITY PRESERVING PROPERTIES FOR SECOND ORDER ODES
}

\author{
T. Nguyen-Ba, T. Giordano, R. Vaillancourt
}

Abstract. New optimal, explicit, $s$-stage Runge-Kutta-Nystrom methods of order $p=3$ to 6 , denoted by $\operatorname{CPRKN}(s, p), p=3,4, \ldots, 6$, that have contractivitypreserving $(\mathrm{CP})$ properties and nonnegative coefficients are constructed for solving the special second-order system of non-stiff ordinary differential equations $y^{\prime \prime}=$ $f(t, y), y\left(t_{0}\right)=y_{0}, y^{\prime}\left(t_{0}\right)=y_{0}^{\prime}$, where $y \in \mathbb{R}^{N}$. Selected $\operatorname{CPRKN}(4,4)$ and $\operatorname{CPRKN}(6,6)$ compare well with Dormand-El-Mikkawy-Prince DEP(4,3)4FM and DEP(6,4)6FM, respectively, in solving standard N-body problems over an interval of 1000 periods on the basis of the relative error of energy (EE) as a function of the number of function evaluations (NFE). The existence of these $\operatorname{CPRKN}(s, p)$ would suggest that they can be combined with Taylor series or CP Hermite-Obrechkoff series methods developed earlier to form new higher order, more efficient methods with contractivitypreserving $(\mathrm{CP})$ properties. The coefficients of $\operatorname{CPRKN}(s, p), p=3,4, \ldots, 6$ are listed in the appendix.

2010 Mathematics Subject Classification: Primary: 65L06; Secondary: 65D05, $65 \mathrm{D} 30$.

Keywords: Contractivity preserving, strong stability preserving, Runge-KuttaNystrom method, second order ordinary differential equations, N-body simulation.

\section{INTRODUCTION}

New optimal, explicit, $s$-stage Runge-Kutta-Nystrom methods of order $p=3$ to 6 , denoted by $\operatorname{CPRKN}(s, p), p=3,4, \ldots, 6$, that has contractivity-preserving (CP) properties and nonnegative coefficients are constructed for solving the special secondorder system of non-stiff ordinary differential equations (ODEs),

$$
y^{\prime \prime}=f(t, y), \quad y\left(t_{0}\right)=y_{0}, \quad y^{\prime}\left(t_{0}\right)=y_{0}^{\prime}, \quad \text { where } \quad{ }^{\prime}=\frac{d}{d t} \quad \text { and } \quad y \in \mathbb{R}^{N} .
$$


T. Nguyen-Ba, T. Giordano, R. Vaillancourt - On RKN formulae ...

We consider now the following form of the solution $y$ to problem (1):

$$
y(t+\Delta t)=y(t)+\Delta t y^{\mathrm{sub}}(t+\Delta t, \Delta t)
$$

where the subformula $y^{\mathrm{sub}}(t+\Delta t, \Delta t)$ (which is the slope $\left.[y(t+\Delta t)-y(t)] / \Delta t\right)$ is the series

$$
y^{\mathrm{sub}}(t+\Delta t, \Delta t)=y^{\prime}(t)+\sum_{m=2}^{\infty} \frac{(\Delta t)^{m-1}}{m !} y^{(m)}(t) .
$$

In our construction of formulae for $y^{\text {sub }}$ defined in (2) and $y^{\prime}$ of $\operatorname{CPRKN}(s, p)$, $p=3,4, \ldots, 6$, we rewrite formulae for $y^{\text {sub }}$ and $y^{\prime}$ as a convex combination of forward Euler (FE) method (approximating $y^{\prime}(t+\Delta t)$ ),

$$
y_{n+1}^{\prime}=y_{n}^{\prime}+\Delta t y_{n}^{\prime \prime} .
$$

If $\mathrm{FE}$ is contractive in a given norm, then formulae for $y^{\text {sub }}$ and $y^{\prime}$ of $\operatorname{CPRKN}(s, p)$ will be contractive as a convex combination of $\mathrm{FE}$ with modified step sizes.

The regions of absolute stability of $\operatorname{CPRKN}(s, p), p=3,4, \ldots, 6$, are derived under the assumption that two solution derivatives, $y^{\prime}$ and $\tilde{y}^{\prime}$, to problem (1) are contractive:

$$
\left\|y^{\prime}(t+\Delta t)-\tilde{y}^{\prime}(t+\Delta t)\right\| \leq\left\|y^{\prime}(t)-\tilde{y}^{\prime}(t)\right\|, \quad \forall \Delta t \geq 0
$$

and the two subformulae $y^{\mathrm{sub}}(t, \Delta t)$ and $\tilde{y}^{\mathrm{sub}}(t, \Delta t)$ defined in (2) are contractive:

$$
\left\|y^{\mathrm{sub}}(t+\Delta t, \Delta t)-\tilde{y}^{\mathrm{sub}}(t+\Delta t, \Delta t)\right\| \leq\left\|y^{\mathrm{sub}}(t, \Delta t)-\tilde{y}^{\mathrm{sub}}(t, \Delta t)\right\|, \quad \forall \Delta t \geq 0,
$$

and

$$
\begin{aligned}
\left\|y^{\prime}(t+\Delta t)-\tilde{y}^{\prime}(t+\Delta t)\right\| & \leq\left\|y^{\mathrm{sub}}(t+\Delta t, \Delta t)-\tilde{y}^{\mathrm{sub}}(t+\Delta t, \Delta t)\right\| \\
& \leq\left\|y^{\prime}(t)-\tilde{y}^{\prime}(t)\right\| \quad \forall \Delta t \geq 0 .
\end{aligned}
$$

We assume that there exists a maximum stepsize $\Delta t_{\mathrm{FE}}$ such that, when $\mathrm{FE}$ is employed with $\Delta t \leq \Delta t_{\mathrm{FE}}, f$ satisfies a discrete analog of (4):

$$
\left\|y_{n+1}^{\prime}-\tilde{y}_{n+1}^{\prime}\right\| \equiv\left\|y_{n}^{\prime}+\Delta t f\left(t_{n}, y_{n}\right)-\left(\tilde{y}_{n}^{\prime}+\Delta t f\left(t_{n}, \tilde{y}_{n}\right)\right)\right\| \leq\left\|y_{n}^{\prime}-\tilde{y}_{n}^{\prime}\right\|,
$$

with

$$
\left\|y_{n}^{\text {sub }}+\Delta t f\left(t_{n}, y_{n}\right)-\left(\tilde{y}_{n}^{\text {sub }}+\Delta t f\left(t_{n}, \tilde{y}_{n}\right)\right)\right\| \leq\left\|y_{n}^{\prime}-\tilde{y}_{n}^{\prime}\right\|,
$$

when $y_{n}^{\prime}$ and $\tilde{y}_{n}^{\prime}$ are replaced by $y_{n}^{\text {sub }}$ and $\tilde{y}_{n}^{\text {sub }}$, respectively, in the two FE formulae in (7), and, when FE is employed with $\Delta t \leq \Delta t_{\mathrm{FE}}, f$ satisfies a discrete analog of (6) :

$$
\left\|y_{n+1}^{\prime}-\tilde{y}_{n+1}^{\prime}\right\| \leq\left\|y_{n+1}^{\mathrm{sub}}-\tilde{y}_{n+1}^{\mathrm{sub}}\right\| \leq\left\|y_{n}^{\prime}-\tilde{y}_{n}^{\prime}\right\| .
$$


T. Nguyen-Ba, T. Giordano, R. Vaillancourt - On RKN formulae ...

Here $y_{n}^{\prime}$ and $\tilde{y}_{n}^{\prime}$ are two derivatives of the numerical solutions generated by FE with different neighbouring starting (or previous) values $y_{0}^{\prime}=y^{\prime}\left(t_{0}\right)$ and $\tilde{y}_{0}^{\prime}=\tilde{y}^{\prime}\left(t_{0}\right)$.

By interpreting $\tilde{y}_{0}^{\prime}$ as a perturbation of $y_{0}^{\prime}$ due to numerical error, we see that contractivity implies that these errors do not grow as they are propagated.

We are interested in a higher-order formula for $y^{\mathrm{sub}}$ of $\operatorname{CPRKN}(s, p), p=3,4, \ldots, 6$, that maintains the contractivity-preserving property

$$
\left\|y_{n+1}^{\mathrm{sub}}-\tilde{y}_{n+1}^{\mathrm{sub}}\right\|\left(\leq\left\|y_{n}^{\prime}-\tilde{y}_{n}^{\prime}\right\|\right) \leq\left\|y_{n}^{\mathrm{sub}}-\tilde{y}_{n}^{\mathrm{sub}}\right\|,
$$

together with a higher-order formula for $y^{\prime}$ that maintains the contractivity-preserving property,

$$
\left\|y_{n+1}^{\prime}-\tilde{y}_{n+1}^{\prime}\right\| \leq\left\|y_{n}^{\prime}-\tilde{y}_{n}^{\prime}\right\|,
$$

for $0 \leq \Delta t \leq \Delta t_{\max }=c \Delta t_{\mathrm{FE}}$ whenever inequality (7) holds. Here $c$, called the contractivity-preserving coefficient (CP coefficient), depends only on the numerical integration method but not on $f$. This definition of the $\mathrm{CP}$ coefficient of $\operatorname{CPRKN}(s, p), p=3,4, \ldots, 6$, follows closely the definition of the strong stability preserving (SSP) coefficient of RK (see [3]).

In [8], similar CP RK methods have been constructed and tested on DETEST problems [7].

The aim of $\operatorname{CPRKN}(s, p), p=3,4, \ldots, 6$, is to maintain simultaneously the CP properties (10) and (11) while achieving higher-order accuracy, perhaps with a modified time-step restriction, measured here with the $\mathrm{CP}$ coefficient $c(\mathrm{CPRKN}(s, p))$ :

$$
\Delta t \leq c(\operatorname{CPRKN}(s, p)) \Delta t_{\mathrm{FE}}
$$

This coefficient describes the ratio of the maximal $\operatorname{CPRKN}(s, p)$ time step to the time step $\Delta t_{\mathrm{FE}}$, for which conditions $(7),(8)$ and (9) hold.

Similar to Huang and Innanen [6], we compare the numerical performance of selected CPRKN(4,4), CPRKN(6, 6), Dormand-El-Mikkawy-Prince DEP(4,3)4FM and $\operatorname{DEP}(6,4) 6 \mathrm{FM}[1]$ on Kepler orbit with eccentricities $e=0.3,0.5,0.7$ over an interval of 1000 periods on the basis of the relative energy error (EE) as a function of the number of function evaluations (NFE). It is seen that $\operatorname{CPRKN}(4,4)$ and CPRKN $(6,6)$ compare well with $\operatorname{DEP}(4,3) 4 \mathrm{FM}$ and $\operatorname{DEP}(6,4) 6 \mathrm{FM}$.

The existence of the new methods $\operatorname{CPRKN}(s, p)$ and the above results would suggest that these CPRKN $(s, p)$ can be combined with Taylor series or HO series methods developed earlier [10] to form new higher order methods with contractivitypreserving $(\mathrm{CP})$ properties and nonnegative coefficients for solving efficiently equations (1).

Section 2 introduces new $\operatorname{CPRKN}(s, p), p=3,4, \ldots, 6$ and the necessary order conditions are listed in Section 3. Section 4 derives $Y_{i}^{\text {sub }}, i=2,3, \ldots, s+1$, of 
T. Nguyen-Ba, T. Giordano, R. Vaillancourt - On RKN formulae ...

$\operatorname{CPRKN}(s, p)$ in contractivity-preserving form (CP form). Section 5 presents $y_{n+1}^{\prime}$ of $\operatorname{CPRKN}(s, p)$ in $\mathrm{CP}$ form for deriving contractivity-preserving (CP) property. Optimizing $\mathrm{CP}$ coefficient $c$ of $\operatorname{CPRKN}(s, p)$ is considered in Section 6. Section 7 describes the intervals of absolute stability of $\operatorname{CPRKN}(4,4)$ and $\operatorname{CPRKN}(6,6)$. In Section 8, numerical results are used to compare $\operatorname{CPRKN}(4,4)$ and $\operatorname{CPRKN}(6,6)$ with Dormand-El-Mikkawy-Prince DEP(4,3)4FM and DEP(6,4)6FM. Coefficients of $\operatorname{CPRKN}(s, p), p=3,4, \ldots, 6$, are listed in Appendix A.

\section{New CPRKN $(s, p)$ With CONTRACtivity-PRESERVing PROPERTIES}

New $\operatorname{CPRKN}(s, p)$ are constructed by the following $(s+1)$ formulae which perform integration from $t_{n}$ to $t_{n+1}$.

Let $\Delta t$ denote the step size. The abscissa vector $\left[c_{1}, c_{2}, \ldots, c_{s}\right]^{T}$ defines the $s$ off-step points $t_{n}+c_{j} \Delta t, j=1,2, \ldots, s$ and $c_{s+1}=1$. In all cases $c_{1}=0$ and, by convention, $c_{1}^{0}=1$. Let $Y_{1}^{\prime \prime}=y_{n}^{\prime \prime}$.

A Hermite-Birkhoff (HB) polynomial is used as stage formula $\mathrm{P}_{i}$ to obtain the stage value $Y_{i}$,

$$
Y_{i}=y_{n}+c_{i} \Delta t y_{n}^{\prime}+\Delta t^{2}\left(\sum_{j=1}^{i-1} \bar{a}_{i j} Y_{j}^{\prime \prime}\right), \quad i=2,3, \ldots, s .
$$

HB polynomials are used as the integration formula IF for $y$ and IF' for $y^{\prime}$ to obtain $y_{n+1}=Y_{3}$ and $y_{n+1}^{\prime}$, respectively, to order $p$,

$$
\begin{aligned}
& y_{n+1}=Y_{s+1}=y_{n}+\Delta t y_{n}^{\prime}+\Delta t^{2}\left(\sum_{j=1}^{s} \bar{b}_{j} Y_{j}^{\prime \prime}\right), \\
& y_{n+1}^{\prime}=y_{n}^{\prime}+\Delta t\left(\sum_{j=1}^{s} b_{j} Y_{j}^{\prime \prime}\right)
\end{aligned}
$$

where $Y_{i}^{\prime \prime}:=f\left(t_{n}+c_{i} \Delta t, Y_{i}\right), i=2,3, \ldots, s$ denotes the stage second derivatives.

Formulae (13)-(15) are the usual form of $\operatorname{CPRKN}(s, p)$.

The defining formulae of $\operatorname{CPRKN}(s, p)$ involve the usual Runge-Kutta-Nystrom (RKN) parameters $c_{i}, \bar{a}_{i, j}, \bar{b}_{j}$ and $b_{j}$.

Thus we can represent $\operatorname{CPRKN}(s, p)$ by its coefficient scheme $(A, \bar{b}, b)$, where $A=\left(\bar{a}_{i, j}\right)$ is a $s \times s$ matrix, $\bar{b}=\left(\bar{b}_{1}, \bar{b}_{2}, \ldots, \bar{b}_{s}\right)^{T}, b=\left(b_{1}, b_{2}, \ldots, b_{s}\right)^{T}$ are two $s$ vector. One can display the coefficient scheme $(A, \bar{b}, b)$, and the $c_{i}$ in the Butcher tableau 


\begin{tabular}{c|cccccc}
$c_{1}$ & & & & & & \\
$c_{2}$ & $\bar{a}_{21}$ & & & & & \\
$c_{3}$ & $\bar{a}_{31}$ & $\bar{a}_{32}$ & & & & \\
$c_{4}$ & $\bar{a}_{41}$ & $\bar{a}_{42}$ & $\bar{a}_{43}$ & & & \\
$\vdots$ & $\vdots$ & & $\ddots$ & $\ddots$ & & \\
$c_{s}$ & $\bar{a}_{s 1}$ & $\bar{a}_{s 2}$ & $\bar{a}_{s 3}$ & $\cdots$ & $\bar{a}_{s, s-1}$ & \\
& $\bar{b}_{1}$ & $\bar{b}_{2}$ & $\bar{b}_{3}$ & $\cdots$ & $\bar{b}_{s-1}$ & $\bar{b}_{s}$ \\
\hline & $b_{1}$ & $b_{2}$ & $b_{3}$ & $\cdots$ & $b_{s-1}$ & $b_{s}$
\end{tabular}

\subsection{Subformulae of $Y_{i}, i=2,3, \ldots, s+1$ of $\operatorname{CPRKN}(s, p)$}

We consider now the following form of $Y_{i}, i=2,3, \ldots, s+1$ with $Y_{s+1}=y_{n+1}$ defined in (13) and (14) respectively,

$$
\begin{aligned}
Y_{i} & =y_{n}+\Delta t\left(Y_{i}^{\mathrm{sub}}\right), \quad i=2,3, \ldots, s, \\
y_{n+1} & =Y_{s+1}=y_{n}+\Delta t\left(y_{n+1}^{\mathrm{sub}}\right),
\end{aligned}
$$

where subformulae $Y_{i}^{\mathrm{sub}}, i=2,3, \ldots, s+1$ are

$$
\begin{aligned}
& Y_{i}^{\mathrm{sub}}=c_{i} y_{n}^{\prime}+\Delta t\left(\sum_{j=1}^{i-1} \bar{a}_{i j} Y_{j}^{\prime \prime}\right), \quad i=2,3, \ldots, s, \\
& y_{n+1}^{\mathrm{sub}}=Y_{s+1}^{\mathrm{sub}}=y_{n}^{\prime}+\Delta t\left(\sum_{j=1}^{s} \bar{b}_{j} Y_{j}^{\prime \prime}\right) .
\end{aligned}
$$

Subformulae (18) and (19) are called the usual form of $Y_{i}^{\text {sub }}, i=2,3, \ldots, s+1$.

\section{Order CONDitions For $\operatorname{CPRKN}(s, p)$}

As in similar search for solvers of (1), we impose the following simplifying assumptions for $Y_{i}$ :

$$
\sum_{j=1}^{i-1} \bar{a}_{i, j}=\frac{c_{i}^{2}}{2}, \quad i=2,3, \ldots, s .
$$

Under the assumption $\overline{b_{i}}=b_{i}\left(1-c_{i}\right), i=1,2, \ldots, s$, there remains the following 11 sets of equations for $y_{n+1}^{\prime}$ to be solved:

$$
\sum_{i=1}^{s} b_{i} \frac{c_{i}^{k}}{k !}=\frac{1}{(k+1) !}, \quad k=0,1, \ldots, p-1,
$$


T. Nguyen-Ba, T. Giordano, R. Vaillancourt - On RKN formulae ...

$$
\begin{aligned}
& \sum_{i=2}^{s} b_{i}\left[\sum_{j=1}^{i-1} \bar{a}_{i j} c_{j}\right]=\frac{1}{4 !}, \\
& \sum_{i=2}^{s} b_{i} \frac{c_{i}}{4}\left[\sum_{j=1}^{i-1} \bar{a}_{i j} c_{j}\right]=\frac{1}{5 !}, \\
& \sum_{i=2}^{s} b_{i}\left[\sum_{j=1}^{i-1} \bar{a}_{i j} \frac{c_{j}^{2}}{2 !}\right]=\frac{1}{5 !}, \\
& \sum_{i=2}^{s} b_{i} \frac{c_{i}^{2}}{4 \times 5}\left[\sum_{j=1}^{i-1} \bar{a}_{i j} c_{j}\right]=\frac{1}{6 !}, \\
& \sum_{i=2}^{s} b_{i} \frac{c_{i}}{5}\left[\sum_{j=1}^{i-1} \bar{a}_{i j} \frac{c_{j}^{2}}{2 !}\right]=\frac{1}{6 !}, \\
& \sum_{i=2}^{s} b_{i}\left[\sum_{j=1}^{i-1} \bar{a}_{i j} \frac{c_{j}^{3}}{3 !}\right]=\frac{1}{6 !}, \\
& \sum_{i=2}^{s} b_{i}\left[\sum_{j=1}^{i-1} \bar{a}_{i j}\left[\sum_{k=1}^{j-1} \bar{a}_{j k} c_{k}\right]\right]=\frac{1}{6 !}, \\
& \sum_{i=2}^{s} b_{i} \frac{c_{i}}{6}\left[\sum_{j=1}^{i-1} \bar{a}_{i j}\left[\sum_{k=1}^{j-1} \bar{a}_{j k} c_{k}\right]\right]=\frac{1}{7 !}, \\
& \sum_{i=2}^{s} b_{i} \frac{c_{i}^{2}}{5 \times 6}\left[\sum_{j=1}^{i-1} \bar{a}_{i j} \frac{c_{j}^{2}}{2 !}\right]=\frac{1}{7 !}, \\
& \sum_{i=2}^{s} b_{i} \frac{c_{i}}{6}\left[\sum_{j=1}^{i-1} \bar{a}_{i j} \frac{c_{j}^{3}}{3 !}\right]=\frac{1}{7 !} . \\
& \sum_{j},
\end{aligned}
$$

Besides conditions (20),

$\operatorname{CPRKN}(2,3)$ satisfies (21) with $k=0,1,2$,

$\operatorname{CPRKN}(3,4)$ satisfies $(21)$ with $k=0,1, \ldots, 4$ and $(22)$,

$\operatorname{CPRKN}(4,4)$ satisfies $(21)$ with $k=0,1, \ldots, 4,(22)$ and (23),

CPRKN $(5,5)$ satisfies $(21)$ with $k=0,1, \ldots, 5,(22)$ to $(27)$,

$\operatorname{CPRKN}(6,6)$ satisfies $(21)$ with $k=0,1, \ldots, 6,(22)$ to $(31)$.

It is to be noted that, in (32), to reduce the norm of the principal local truncation error coefficients, some additional order conditions associated with $(\Delta t)^{p+1}$ are 
satisfied.

\section{From modified CONTRACTIVITY-PRESERVing (CP) FORM TO CANONICAL CP FORM OF $Y_{i}^{\mathrm{SUB}}, i=2,3, \ldots, s+1$ of $\operatorname{CPRKN}(s, p)$}

Gottlieb, Ketcheson and Shu presented canonical Shu-Osher forms in compact vector notation for RK methods (see [4, Section 3.1-3.4] for details).

Our construction of the canonical CP form (similar to the canonical Shu-Osher form for RK methods) of $Y_{i}^{\mathrm{sub}}, i=2,3, \ldots, s+1$ of $\operatorname{CPRKN}(s, p)$ proceeds in three steps in Subsections 4.1-4.3.

\subsection{Contractivity preserving of $Y_{i}^{\text {sub }}, i=2,3, \ldots, s+1$ of $\operatorname{CPRKN}(s, p)$}

Similar to the Shu-Osher form of RK methods [12], equations (18)-(19) of $Y_{i}^{\text {sub }}$, $i=2,3, \ldots, s$ and $Y_{s+1}^{\mathrm{sub}}=y_{n+1}^{\mathrm{sub}}$, respectively, can be written in the form,

$$
\begin{aligned}
& Y_{i}^{\text {sub }}=\bar{\alpha}_{i 1} y_{n}^{\prime}+\Delta t \bar{\beta}_{i 1} y_{n}^{\prime \prime}+\left[\sum_{j=1}^{i-1} \bar{\alpha}_{i j} Y_{j}^{\text {sub }}+\Delta t \bar{\beta}_{i j} Y_{j}^{\prime \prime}\right], \quad i=2,3, \ldots, s+1, \\
& y_{n+1}^{\text {sub }}=Y_{s+1}^{\text {sub }},
\end{aligned}
$$

with consistency conditions:

$$
\bar{\alpha}_{i 1}+\sum_{j=2}^{i-1} \bar{\alpha}_{i j} c_{j}=c_{i}, \quad i=2,3, \ldots, s+1 .
$$

Form (33) is called the contractivity preserving form (CP form) (similar to ShuOsher form for RK methods [4]) of $Y_{i}^{\text {sub }}, i=2,3, \ldots, s+1$ of $\operatorname{CPRKN}(s, p)$.

By setting $\bar{v}_{i}=\bar{\alpha}_{i 1}$ and $\bar{w}_{i}=\bar{\beta}_{i 1}, i=2,3, \ldots, s+1$, in (33), we have the modified $C P$ form of $Y_{i}^{\text {sub }}, i=2,3, \ldots, s+1$ of $\operatorname{CPRKN}(s, p)$ :

$$
\begin{aligned}
& Y_{i}^{\mathrm{sub}}=\bar{v}_{i} y_{n}^{\prime}+\Delta t \bar{w}_{i} y_{n}^{\prime \prime}+\left[\sum_{j=2}^{i-1} \bar{\alpha}_{i j} Y_{j}^{\mathrm{sub}}+\Delta t \bar{\beta}_{i j} Y_{j}^{\prime \prime}\right], \quad i=2,3, \ldots, s+1, \\
& y_{n+1}^{\mathrm{sub}}=Y_{s+1}^{\mathrm{sub}} .
\end{aligned}
$$

We can rearrange the stage values $Y_{i}^{\mathrm{sub}}, i=2,3, \ldots, s+1$, in (35) as follows:

$$
\begin{aligned}
Y_{i}^{\mathrm{sub}}=\bar{v}_{i}\left[y_{n}^{\prime}+\Delta t \frac{\bar{w}_{i}}{\bar{v}_{i}} y_{n}^{\prime \prime}\right]+\sum_{j=2}^{i-1} \bar{\alpha}_{i j}\left(Y_{j}^{\mathrm{sub}}+\Delta t \frac{\bar{\beta}_{i j}}{\bar{\alpha}_{i j}} Y_{j}^{\prime \prime}\right) & \\
& i=2,3, \ldots, s+1,
\end{aligned}
$$


with consistency conditions:

$$
\bar{v}_{i}+\sum_{j=2}^{i-1} \bar{\alpha}_{i j} c_{j}=c_{i}, \quad i=2,3, \ldots, s+1 .
$$

Thus we obtain the difference $Y_{i}^{\mathrm{sub}}-\widetilde{Y}_{i}^{\text {sub }}, i=2,3, \ldots, s+1$, from (36) as follows:

$$
\begin{aligned}
Y_{i}^{\mathrm{sub}}-\tilde{Y}_{i}^{\mathrm{sub}} & =\bar{v}_{i}\left[\left(y_{n}^{\prime}+\Delta t \frac{\bar{w}_{i}}{\bar{v}_{i}} y_{n}^{\prime \prime}\right)-\left(\tilde{y}_{n}^{\prime}+\Delta t \frac{\bar{w}_{i}}{\bar{v}_{i}} \tilde{y}_{n}^{\prime \prime}\right)\right] \\
& +\sum_{j=2}^{i-1} \bar{\alpha}_{i j}\left[\left(Y_{j}^{\mathrm{sub}}+\Delta t \frac{\bar{\beta}_{i j}}{\bar{\alpha}_{i j}} Y_{j}^{\prime \prime}\right)-\left(\tilde{Y}_{j}^{\mathrm{sub}}+\Delta t \frac{\bar{\beta}_{i j}}{\bar{\alpha}_{i j}} \widetilde{Y}_{j}^{\prime \prime}\right)\right], \quad i=2,3, \ldots, s+1 .
\end{aligned}
$$

Provided all the coefficients of (36) are nonnegative, the following straightforward extension of a result presented in $[2,5]$ holds.

Theorem 1. If $f$ satisfies conditions (7), (8) and (9) of the FE method, then $y_{n+1}^{\text {sub }}=Y_{s+1}^{\text {sub }}$ of the $\operatorname{CPRKN}(s, p)$ method (36) satisfies the CP property

$$
\left\|y_{n+1}^{\mathrm{sub}}-\tilde{y}_{n+1}^{\mathrm{sub}}\right\| \leq\left\|y_{n}^{\prime}-\tilde{y}_{n}^{\prime}\right\| \leq\left\|y_{n}^{\mathrm{sub}}-\tilde{y}_{n}^{\mathrm{sub}}\right\|
$$

provided

$$
\Delta t \leq c_{\text {feasible }} \Delta t_{\mathrm{FE}}
$$

where the feasible CP coefficient, $c_{\text {feasible, }}$ is the minimum of the following numbers:

$$
\frac{\bar{\alpha}_{i j}}{\bar{\beta}_{i j}}, \quad j=2,3, \ldots, i-1, \text { and } \quad \frac{\bar{v}_{i}}{\bar{w}_{i}}, \quad i=2,3, \ldots, s+1,
$$

under the assumption that all coefficients of (36) are nonnegative, with the convention that the ratios $a / 0=+\infty$, and the ratios $0 / 0$ are ignored.

Proof. The difference $Y_{i}^{\text {sub }}-\widetilde{Y}_{i}^{\text {sub }}$ of $\operatorname{CPRKN}(s, p)$ can be rewritten as a convex combination of the two terms on the right-hand side of (38). Thus, by the convexity of the norm $\|\cdot\|$, we have

$$
\begin{aligned}
\| Y_{i}^{\mathrm{sub}} & -\tilde{Y}_{i}^{\mathrm{sub}}\left\|\leq \bar{v}_{i}\right\|\left(y_{n}^{\prime}+\Delta t \frac{\bar{w}_{i}}{\bar{v}_{i}} y_{n}^{\prime \prime}\right)-\left(\tilde{y}_{n}^{\prime}+\Delta t \frac{\bar{w}_{i}}{\bar{v}_{i}} \tilde{y}_{n}^{\prime \prime}\right) \| \\
& +\sum_{j=2}^{i-1} \bar{\alpha}_{i j}\left\|\left(Y_{j}^{\mathrm{sub}}+\Delta t \frac{\bar{\beta}_{i j}}{\bar{\alpha}_{i j}} Y_{j}^{\prime \prime}\right)-\left(\tilde{Y}_{j}^{\mathrm{sub}}+\Delta t \frac{\bar{\beta}_{i j}}{\bar{\alpha}_{i j}} \tilde{Y}_{j}^{\prime \prime}\right)\right\|, \quad i=2,3, \ldots, s+1 .
\end{aligned}
$$


We present a proof by induction on $i$. When $i=2$, the right-hand side of (40) has the following upper bound, since $\frac{\bar{w}_{2}}{\bar{v}_{2}} \Delta t \leq \frac{\Delta t}{c_{\text {feasible }}} \leq \Delta t_{\mathrm{FE}}$,

$$
\begin{aligned}
& \bar{v}_{2}\left\|\left(y_{n}^{\prime}+\Delta t \frac{\bar{w}_{2}}{\bar{v}_{2}} y_{n}^{\prime \prime}\right)-\left(\tilde{y}_{n}^{\prime}+\Delta t \frac{\bar{w}_{2}}{\bar{v}_{2}} \tilde{y}_{n}^{\prime \prime}\right)\right\| \\
& \quad \leq \bar{v}_{2}\left\|y_{n}^{\prime}-\tilde{y}_{n}^{\prime}\right\| \quad \text { by }(7), \\
& \quad \leq c_{2}\left\|y_{n}^{\prime}-\tilde{y}_{n}^{\prime}\right\| \quad \text { by }(37) \text { with } i=2, \\
& \quad \leq c_{2}\left\|y_{n}^{\text {sub }}-\tilde{y}_{n}^{\text {sub }}\right\| \quad \text { by }(9) .
\end{aligned}
$$

Suppose now that, for $k=2,3, \ldots, i-1,\left\|Y_{k}^{\text {sub }}-\widetilde{Y}_{k}^{\text {sub }}\right\|$ of (40) has the following upper bound,

$$
\left\|Y_{k}^{\mathrm{sub}}-\tilde{Y}_{k}^{\mathrm{sub}}\right\| \leq c_{k}\left\|y_{n}^{\prime}-\tilde{y}_{n}^{\prime}\right\| \leq c_{k}\left\|y_{n}^{\mathrm{sub}}-\tilde{y}_{n}^{\mathrm{sub}}\right\| .
$$

Then, for $k=i,\left\|Y_{i}^{\mathrm{sub}}-\tilde{Y}_{i}^{\mathrm{sub}}\right\|$ of (40) has the following upper bound, since $\frac{\bar{\beta}_{i j}}{\bar{\alpha}_{i j}} \Delta t \leq$ $\frac{\Delta t}{c_{\text {feasible }}} \leq \Delta t_{\mathrm{FE}}$

$$
\begin{aligned}
& \left\|Y_{i}^{\mathrm{sub}}-\tilde{Y}_{i}^{\mathrm{sub}}\right\| \leq \bar{v}_{i}\left\|\left(y_{n}^{\prime}+\Delta t \frac{\bar{w}_{i}}{\bar{v}_{i}} y_{n}^{\prime \prime}\right)-\left(\tilde{y}_{n}^{\prime}+\Delta t \frac{\bar{w}_{i}}{\bar{v}_{i}} \tilde{y}_{n}^{\prime \prime}\right)\right\| \\
& \quad \sum_{j=2}^{i-1} \bar{\alpha}_{i j}\left\|\left(Y_{j}^{\text {sub }}+\Delta t \frac{\bar{\beta}_{i j}}{\bar{\alpha}_{i j}} Y_{j}^{\prime \prime}\right)-\left(\tilde{Y}_{j}^{\text {sub }}+\Delta t \frac{\bar{\beta}_{i j}}{\bar{\alpha}_{i j}} \tilde{Y}_{j}^{\prime \prime}\right)\right\| \\
& \leq \bar{v}_{i}\left\|y_{n}^{\prime}-\tilde{y}_{n}^{\prime}\right\|+\sum_{j=2}^{i-1} \bar{\alpha}_{i j}\left\|\left(Y_{j}^{\text {sub }}+\frac{\Delta t}{c_{\text {feasible }}} Y_{j}^{\prime \prime}\right)-\left(\tilde{Y}_{j}^{\text {sub }}+\frac{\Delta t}{c_{\text {feasible }}} \tilde{Y}_{j}^{\prime \prime}\right)\right\| \\
& \leq \bar{v}_{i}\left\|y_{n}^{\prime}-\tilde{y}_{n}^{\prime}\right\|+\sum_{j=2}^{i-1} \bar{\alpha}_{i j}\left\|Y_{j}^{\text {sub }}-\tilde{Y}_{j}^{\text {sub }}\right\| \quad \text { by }(8) \text { and }(9) \\
& \leq \bar{v}_{i}\left\|y_{n}^{\prime}-\tilde{y}_{n}^{\prime}\right\|+\sum_{j=2}^{i-1} \bar{\alpha}_{i j} c_{j}\left\|y_{n}^{\prime}-\tilde{y}_{n}^{\prime}\right\| \quad \text { by }(42) \\
& \leq c_{i}\left\|y_{n}^{\prime}-\tilde{y}_{n}^{\prime}\right\| \quad \text { by }(37), \\
& \leq c_{i}\left\|y_{n}^{\text {sub }}-\tilde{y}_{n}^{\text {sub }}\right\| \text { by }(9) .
\end{aligned}
$$

Thus, we have, from (44) and (45), the following upper bound of $\left\|Y_{i}^{\text {sub }}-\tilde{Y}_{i}^{\text {sub }}\right\|$,

$$
\begin{aligned}
\left\|Y_{i}^{\mathrm{sub}}-\tilde{Y}_{i}^{\mathrm{sub}}\right\| & \leq c_{i}\left\|y_{n}^{\prime}-\tilde{y}_{n}^{\prime}\right\|, \\
& \leq c_{i}\left\|y_{n}^{\mathrm{sub}}-\tilde{y}_{n}^{\mathrm{sub}}\right\|, \quad i=2,3, \ldots, s+1,
\end{aligned}
$$


and, naturally,

$$
\begin{aligned}
\left\|Y_{i}^{\mathrm{sub}}-\tilde{Y}_{i}^{\mathrm{sub}}\right\| & \leq\left\|y_{n}^{\prime}-\tilde{y}_{n}^{\prime}\right\|, \\
& \leq\left\|y_{n}^{\mathrm{sub}}-\tilde{y}_{n}^{\mathrm{sub}}\right\|, \quad i=2,3, \ldots, s+1 .
\end{aligned}
$$

In particular, this yields $\left\|y_{n+1}^{\mathrm{sub}}-\tilde{y}_{n+1}^{\mathrm{sub}}\right\| \leq\left\|y_{n}^{\prime}-\tilde{y}_{n}^{\prime}\right\| \leq\left\|y_{n}^{\mathrm{sub}}-\tilde{y}_{n}^{\mathrm{sub}}\right\|$.

It is to be noted here that each representation (36) of $Y_{i}^{\text {sub }}, i=2,3, \ldots, s+$ 1 (to obtain $Y_{s+1}=y_{n+1}$ to order $p$ ) with coefficients $\bar{v}_{i}, \bar{w}_{i}, \bar{\alpha}_{i j}$ and $\bar{\beta}_{i j}$, will produce a feasible CP coefficient, $c_{\text {feasible }}$, defined in Theorem 1 and feasible $Y_{i}^{\text {sub }}$, $i=2,3, \ldots, s+1$ of $\operatorname{CPRKN}(s, p)$ in modified CP form (36). What we really want is not merely feasible $Y_{i}^{\text {sub }}, i=2,3, \ldots, s+1$ of method $\operatorname{CPRKN}(s, p)$ in CP form but best $Y_{i}^{\text {sub }}, i=2,3, \ldots, s+1$. This question will be considered in Section 6 .

Transforming the usual form (18)-(19) into the modified CP form (35) of $Y_{i}^{\text {sub }}$, $i=2,3, \ldots, s+1$ of $\operatorname{CPRKN}(s, p)$ and vice versa will be considered in Subsection 4.2.

\subsection{Vector notation of $Y_{i}^{\text {sub }}, i=2,3, \ldots, s+1$ of $\operatorname{CPRKN}(s, p)$}

Vector and matrix notation will help represent $Y_{i}^{\text {sub }}, i=2,3, \ldots, s+1$ of $\operatorname{CPRKN}(s, p)$ in canonical CP form.

We define $(s+1)$-vectors

$$
\overline{\boldsymbol{v}}=\left[0, \bar{v}_{2}, \bar{v}_{3}, \ldots, \bar{v}_{s+1}\right]^{T}, \quad \overline{\boldsymbol{w}}=\left[0, \bar{w}_{2}, \bar{w}_{3}, \ldots, \bar{w}_{s+1}\right]^{T},
$$

strictly lower triangular real matrices $\overline{\boldsymbol{\alpha}}, \overline{\boldsymbol{\beta}} \in \mathbb{R}^{(s+1) \times(s+1)}$,

$$
\overline{\boldsymbol{\alpha}}=\left\{\bar{\alpha}_{i j}\right\}, \quad \overline{\boldsymbol{\beta}}=\left\{\bar{\beta}_{i j}\right\} .
$$

The components $\bar{v}_{i}, \bar{w}_{i}, \bar{\alpha}_{i j}, \bar{\beta}_{i j}$ come from the modified CP form (35) of $Y_{i}^{\text {sub }}$, $i=2,3, \ldots, s+1$ of $\operatorname{CPRKN}(s, p)$.

Moreover,

$$
\boldsymbol{Y}^{\mathrm{sub}}=\left[0, Y_{2}^{\mathrm{sub}}, Y_{3}^{\mathrm{sub}}, \ldots, Y_{s+1}^{\mathrm{sub}}\right]^{T}, \quad \boldsymbol{F}=\left[0, Y_{2}^{\prime \prime}, Y_{3}^{\prime \prime}, \ldots, Y_{s+1}^{\prime \prime}\right]^{T},
$$

with the following $N$-vectors: $Y_{j}^{\text {sub }}, Y_{j}, Y_{j}^{\prime \prime}$ for $j=1,2,3, \ldots, s+1, Y_{1}=y_{n}, Y_{1}^{\prime \prime}=$ $y_{n}^{\prime \prime}=f_{n}, Y_{s+1}^{\mathrm{sub}}=y_{n+1}^{\mathrm{sub}}$ and $Y_{s+1}^{\prime \prime}=y_{n+1}^{\prime \prime}=f_{n+1}$. 
T. Nguyen-Ba, T. Giordano, R. Vaillancourt - On RKN formulae ...

\subsection{1. $\quad$ Modified CP form of $Y_{i}^{\mathrm{sub}}, i=2,3, \ldots, s+1$ in vector notation}

Using the above notation, we rewrite the modified CP form (35) of $Y_{j}^{\text {sub }}, i=$ $2,3, \ldots, s+1$ of $\operatorname{CPRKN}(s, p)$ in vector notation:

$$
\begin{aligned}
& \boldsymbol{Y}^{\mathrm{sub}}=\overline{\boldsymbol{v}}\left(y_{n}^{\prime}\right)^{T}+\Delta t \overline{\boldsymbol{w}}\left(y_{n}^{\prime \prime}\right)^{T}+\overline{\boldsymbol{\alpha}} \boldsymbol{Y}^{\mathrm{sub}}+\Delta t \overline{\boldsymbol{\beta}} \boldsymbol{F}, \\
& y_{n+1}^{\mathrm{sub}}=Y_{s+1}^{\mathrm{sub}},
\end{aligned}
$$

with consistency conditions (37) written in vector form,

$$
\overline{\boldsymbol{v}}+\overline{\boldsymbol{\alpha}} \boldsymbol{e}_{s+1}=\boldsymbol{e}_{s+1},
$$

where

$$
\boldsymbol{e}_{s+1}=\left[0, c_{2}, c_{3}, \ldots, c_{s+1}\right]^{T} \in \mathbb{R}^{s+1} .
$$

It is to be noted that, by setting the first row of matrices $\boldsymbol{Y}^{\text {sub }}, \boldsymbol{F}$ equal to zero, $\bar{\alpha}_{i 1}$ and $\bar{\beta}_{i 1}, i=2,3, \ldots, s+1$ are not used in formulae (51) and are replaced by $\bar{v}_{i}$ and $\bar{w}_{i}, i=2,3, \ldots, s+1$, respectively.

\subsubsection{Usual form of $Y_{i}^{\mathrm{sub}}, i=2,3, \ldots, s+1$ in vector notation}

If $\overline{\boldsymbol{\alpha}}=\mathbf{0}$, then the modified CP form (51) becomes

$$
\begin{aligned}
\boldsymbol{Y}^{\text {sub }} & =\overline{\boldsymbol{v}}\left(y_{n}^{\prime}\right)^{T}+\Delta t \overline{\boldsymbol{w}}\left(y_{n}^{\prime \prime}\right)^{T}+\Delta t \overline{\boldsymbol{\beta}} \boldsymbol{F}, \\
y_{n+1}^{\text {sub }} & =Y_{s+1}^{\text {sub }},
\end{aligned}
$$

which is the usual form. The elements $\overline{\boldsymbol{v}}, \overline{\boldsymbol{w}}, \overline{\boldsymbol{\beta}}$ of $(54)$ are then denoted as $\overline{\boldsymbol{v}}_{0}, \overline{\boldsymbol{w}}_{0}$, $\overline{\boldsymbol{\beta}}_{0}$ respectively, and hence the usual form (54) can be rewritten as

$$
\begin{aligned}
\boldsymbol{Y}^{\mathrm{sub}} & =\overline{\boldsymbol{v}}_{0}\left(y_{n}^{\prime}\right)^{T}+\Delta t \overline{\boldsymbol{w}}_{0}\left(y_{n}^{\prime \prime}\right)^{T}+\Delta t \overline{\boldsymbol{\beta}}_{0} \boldsymbol{F}, \\
y_{n+1}^{\mathrm{sub}} & =Y_{s+1}^{\text {sub }},
\end{aligned}
$$

with the consistency condition,

$$
\overline{\boldsymbol{v}}_{0}=\boldsymbol{e}_{s+1},
$$

where $\boldsymbol{e}_{s+1}$ is defined in (53).

To find the relation between the CP form coefficients and the usual form coefficients, we can solve (51) for $\boldsymbol{Y}^{\text {sub }}$ since $\boldsymbol{I}-\overline{\boldsymbol{\alpha}}$ is invertible because the matrix $\overline{\boldsymbol{\alpha}}$ is strictly lower triangular,

$$
\boldsymbol{Y}^{\mathrm{sub}}=(\boldsymbol{I}-\overline{\boldsymbol{\alpha}})^{-1} \overline{\boldsymbol{v}}\left(y_{n}^{\prime}\right)^{T}+\Delta t(\boldsymbol{I}-\overline{\boldsymbol{\alpha}})^{-1} \overline{\boldsymbol{w}}\left(y_{n}^{\prime \prime}\right)^{T}+\Delta t(\boldsymbol{I}-\overline{\boldsymbol{\alpha}})^{-1} \overline{\boldsymbol{\beta}} \boldsymbol{F} .
$$


T. Nguyen-Ba, T. Giordano, R. Vaillancourt - On RKN formulae ...

Comparing (57) with (55), we have the following relations between the generalized $\mathrm{CP}$ form coefficients and the usual form coefficients,

$$
\overline{\boldsymbol{v}}_{0}=(\boldsymbol{I}-\overline{\boldsymbol{\alpha}})^{-1} \overline{\boldsymbol{v}}, \quad \overline{\boldsymbol{w}}_{0}=(\boldsymbol{I}-\overline{\boldsymbol{\alpha}})^{-1} \overline{\boldsymbol{w}}, \quad \overline{\boldsymbol{\beta}}_{0}=(\boldsymbol{I}-\overline{\boldsymbol{\alpha}})^{-1} \overline{\boldsymbol{\beta}} .
$$

These relations allow a simple transformation of the vectors and matrices $\overline{\boldsymbol{v}}, \overline{\boldsymbol{w}}, \overline{\boldsymbol{\beta}}$ of a CP form into $\overline{\boldsymbol{v}}_{0}, \overline{\boldsymbol{w}}_{0}, \overline{\boldsymbol{\beta}}_{0}$ of a usual form and vice versa.

In fact, the form (55) is the usual form (18) and (19) with

$$
\begin{aligned}
\overline{\boldsymbol{v}}_{0}= & {\left[0, c_{2}, c_{3}, \ldots, c_{s+1}\right]^{T}, \quad \overline{\boldsymbol{w}}_{0}=\left[0, \bar{a}_{21}, \bar{a}_{31}, \ldots, \bar{b}_{1}\right]^{T}, } \\
\overline{\boldsymbol{\beta}}_{0}= & {\left[\begin{array}{ccccccc}
0 & 0 & 0 & 0 & \ldots & 0 & 0 \\
\bar{a}_{21} & 0 & 0 & 0 & \ldots & 0 & 0 \\
\bar{a}_{31} & \bar{a}_{32} & 0 & 0 & \ldots & 0 & 0 \\
\bar{a}_{41} & \bar{a}_{42} & \bar{a}_{43} & 0 & \ldots & 0 & 0 \\
\vdots & \vdots & & \ddots & \ddots & & \vdots \\
\bar{a}_{s 1} & \bar{a}_{s 2} & \bar{a}_{s 3} & \cdots & \bar{a}_{s, s-1} & 0 & 0 \\
\bar{b}_{1} & \bar{b}_{2} & \bar{b}_{3} & \cdots & \bar{b}_{s-1} & \bar{b}_{s} & 0
\end{array}\right] }
\end{aligned}
$$

\subsection{Canonical CP form of $\boldsymbol{Y}^{\mathrm{sub}}$ of $\operatorname{CPRKN}(s, p)$ in vector notation}

To find the $\mathrm{CP}$ coefficient of $\operatorname{CPRKN}(s, p)$, it is useful to consider a particular modified CP form (51) where the elements of the matrices $\overline{\boldsymbol{\alpha}}$ and $\overline{\boldsymbol{\beta}}$ satisfy the same ratio $r=\frac{\bar{\alpha}_{i j}}{\bar{\beta}_{i j}}$ for every $i, j, i=3,4, \ldots, s+1$ and $j=2,3, \ldots, i-1$, such that $\bar{\beta}_{i j} \neq 0$, or, in vector notation,

$$
\overline{\boldsymbol{\alpha}}_{r}=r \overline{\boldsymbol{\beta}}_{r} .
$$

Substituting this relation into (58), we can solve for $\overline{\boldsymbol{\beta}}_{r}$ in terms of $\overline{\boldsymbol{\beta}}_{0}$ and $r$.

First, we have

$$
\left(\boldsymbol{I}-r \overline{\boldsymbol{\beta}}_{r}\right)^{-1} \overline{\boldsymbol{\beta}}_{r}=\overline{\boldsymbol{\beta}}_{0} \quad \Leftrightarrow \quad \overline{\boldsymbol{\beta}}_{r}=\overline{\boldsymbol{\beta}}_{0}-r \overline{\boldsymbol{\beta}}_{r} \overline{\boldsymbol{\beta}}_{0} \quad \Leftrightarrow \quad \overline{\boldsymbol{\beta}}_{r}\left(\boldsymbol{I}+r \overline{\boldsymbol{\beta}}_{0}\right)=\overline{\boldsymbol{\beta}}_{0} .
$$

Then, since $\boldsymbol{I}+r \overline{\boldsymbol{\beta}}_{0}$ is invertible, the coefficients of the CP form (51) are given in terms of the coefficients of the usual form (55) by the expressions

$$
\begin{array}{ll}
\overline{\boldsymbol{v}}_{r}=\left(\boldsymbol{I}+r \overline{\boldsymbol{\beta}}_{0}\right)^{-1} \overline{\boldsymbol{v}}_{0} & =\left(\boldsymbol{I}-\overline{\boldsymbol{\alpha}}_{r}\right) \overline{\boldsymbol{v}}_{0}, \\
\overline{\boldsymbol{w}}_{r}=\left(\boldsymbol{I}+r \overline{\boldsymbol{\beta}}_{0}\right)^{-1} \overline{\boldsymbol{w}}_{0} & =\left(\boldsymbol{I}-\overline{\boldsymbol{\alpha}}_{r}\right) \overline{\boldsymbol{w}}_{0}, \\
\overline{\boldsymbol{\alpha}}_{r}=r \overline{\boldsymbol{\beta}}_{r}=r \overline{\boldsymbol{\beta}}_{0}\left(\boldsymbol{I}+r \overline{\boldsymbol{\beta}}_{0}\right)^{-1} & =r \overline{\boldsymbol{\beta}}_{0}\left(\boldsymbol{I}-\overline{\boldsymbol{\alpha}}_{r}\right), \\
\overline{\boldsymbol{\beta}}_{r}=\overline{\boldsymbol{\beta}}_{0}\left(\boldsymbol{I}+r \overline{\boldsymbol{\beta}}_{0}\right)^{-1} & =\overline{\boldsymbol{\beta}}_{0}\left(\boldsymbol{I}-\overline{\boldsymbol{\alpha}}_{r}\right),
\end{array}
$$


where the identity $\left(\boldsymbol{I}-\overline{\boldsymbol{\alpha}}_{r}\right)=\left(\boldsymbol{I}+r \overline{\boldsymbol{\beta}}_{0}\right)^{-1}$ follows from

$$
\left(\boldsymbol{I}-\overline{\boldsymbol{\alpha}}_{r}\right)\left(\boldsymbol{I}+r \overline{\boldsymbol{\beta}}_{0}\right)=\left(\boldsymbol{I}-r \overline{\boldsymbol{\beta}}_{r}\right)\left(\boldsymbol{I}+r \overline{\boldsymbol{\beta}}_{0}\right)=\boldsymbol{I}+r \overline{\boldsymbol{\beta}}_{0}-r \overline{\boldsymbol{\beta}}_{r}-r^{2} \overline{\boldsymbol{\beta}}_{r} \overline{\boldsymbol{\beta}}_{0}=\boldsymbol{I},
$$

since $r \overline{\boldsymbol{\beta}}_{r}=r \overline{\boldsymbol{\beta}}_{0}-r^{2} \overline{\boldsymbol{\beta}}_{r} \overline{\boldsymbol{\beta}}_{0}$.

It is to be noted that using (58) and (65), $\overline{\boldsymbol{\beta}}_{r}$ can then be written as

$$
\overline{\boldsymbol{\beta}}_{r}=\overline{\boldsymbol{\beta}}_{0}\left(\boldsymbol{I}+r \overline{\boldsymbol{\beta}}_{0}\right)^{-1}=\overline{\boldsymbol{\beta}}_{0}\left(\boldsymbol{I}-\overline{\boldsymbol{\alpha}}_{r}\right)=\left(\boldsymbol{I}-\overline{\boldsymbol{\alpha}}_{r}\right) \overline{\boldsymbol{\beta}}_{0}=\left(\boldsymbol{I}+r \overline{\boldsymbol{\beta}}_{0}\right)^{-1} \overline{\boldsymbol{\beta}}_{0} .
$$

As in [4], we shall refer to the form given by the coefficients (62)-(65), as the canonical $C P$ form of $\operatorname{CPRKN}(s, p)$ :

$$
\boldsymbol{Y}^{\mathrm{sub}}=\overline{\boldsymbol{v}}_{r}\left(y_{n}^{\prime}\right)^{T}+\Delta t \overline{\boldsymbol{w}}_{r}\left(y_{n}^{\prime \prime}\right)^{T}+\overline{\boldsymbol{\alpha}}_{r} \boldsymbol{Y}^{\mathrm{sub}}+\Delta t \overline{\boldsymbol{\beta}}_{r} \boldsymbol{F},
$$

which can be written solely in terms of the vectors and matrices $\overline{\boldsymbol{v}}_{0}, \overline{\boldsymbol{w}}_{0}, \overline{\boldsymbol{\beta}}_{\mathbf{0}}$ of the usual form (55),

$$
\begin{aligned}
\boldsymbol{Y}^{\mathrm{sub}}= & \left(\boldsymbol{I}+r \overline{\boldsymbol{\beta}}_{0}\right)^{-1} \overline{\boldsymbol{v}}_{0}\left(y_{n}^{\prime}\right)^{T}+\Delta t\left(\boldsymbol{I}+r \overline{\boldsymbol{\beta}}_{0}\right)^{-1} \overline{\boldsymbol{w}}_{0}\left(y_{n}^{\prime \prime}\right)^{T} \\
& +r \overline{\boldsymbol{\beta}}_{0}\left(\boldsymbol{I}+r \overline{\boldsymbol{\beta}}_{0}\right)^{-1} \boldsymbol{Y}^{\mathrm{sub}}+\Delta t \overline{\boldsymbol{\beta}}_{0}\left(\boldsymbol{I}+r \overline{\boldsymbol{\beta}}_{0}\right)^{-1} \boldsymbol{F} .
\end{aligned}
$$

The canonical CP form (67) and the form (68) will allow us to formulate simply the optimization problem considered in Section 6.

Using (66) and (68), we obtain

$$
\boldsymbol{Y}^{\mathrm{sub}}=\left(\boldsymbol{I}+r \overline{\boldsymbol{\beta}}_{0}\right)^{-1}\left[\overline{\boldsymbol{v}}_{0}\left(y_{n}^{\prime}\right)^{T}+\Delta t \overline{\boldsymbol{w}}_{0}\left(y_{n}^{\prime \prime}\right)^{T}+\overline{\boldsymbol{\beta}}_{0}\left(r \boldsymbol{Y}^{\mathrm{sub}}+\Delta t \boldsymbol{F}\right)\right] .
$$

Here consistency requires that

$$
\left(\boldsymbol{I}+r \overline{\boldsymbol{\beta}}_{0}\right)^{-1} \overline{\boldsymbol{v}}_{0}+r\left(\boldsymbol{I}+r \overline{\boldsymbol{\beta}}_{0}\right)^{-1} \overline{\boldsymbol{\beta}}_{0} \boldsymbol{e}_{s+1}=\boldsymbol{e}_{s+1},
$$

where $\boldsymbol{e}_{s+1}$ is defined in (53). Condition (70) is equivalent to the consistency condition $(52)$.

Note that the vectorial usual form (55), with $\overline{\boldsymbol{v}}_{0}, \overline{\boldsymbol{w}}_{0}, \overline{\boldsymbol{\beta}}_{0}$, corresponds to the canonical CP form (67) or (69) with $r=0$.

Relations (62)-(65) will enable us to obtain simply the vectors and matrices of a canonical CP form (67) from those of a usual form (55) and vice versa.

To simplify notation, in the following theorem, the ratio $r=\frac{\bar{\alpha}_{i j}}{\bar{\beta}_{i j}}$ which is the same for every $i, j, i=3,4, \ldots, s+1$ and $j=2,3, \ldots, i-1$, becomes a feasible $\mathrm{CP}$ coefficient of $\operatorname{CPRKN}(s, p)$. Hence, $r$ must satisfy the conditions:

$$
r \leq \frac{\bar{v}_{i}}{\bar{w}_{i}}, \quad i=2,3, \ldots, s+1 .
$$

Therefore, the following slight modification of Theorem 1 holds. 
T. Nguyen-Ba, T. Giordano, R. Vaillancourt - On RKN formulae ...

Theorem 2. If $f$ satisfies conditions (7), (8) and (9) of the FE method, then $y_{n+1}^{\text {sub }}$ of $\operatorname{CPRKN}(s, p)$ method (36) satisfies the CP property

$$
\left\|y_{n+1}^{\mathrm{sub}}-\tilde{y}_{n+1}^{\mathrm{sub}}\right\| \leq\left\|y_{n}^{\prime}-\tilde{y}_{n}^{\prime}\right\| \leq\left\|y_{n}^{\mathrm{sub}}-\tilde{y}_{n}^{\mathrm{sub}}\right\|
$$

provided

$$
\Delta t \leq c\left(\overline{\boldsymbol{v}}_{r}, \overline{\boldsymbol{w}}_{r}, \overline{\boldsymbol{\alpha}}_{r}, \overline{\boldsymbol{\beta}}_{r}\right) \Delta t_{\mathrm{FE}},
$$

where

- $c\left(\overline{\boldsymbol{v}}_{r}, \overline{\boldsymbol{w}}_{r}, \overline{\boldsymbol{\alpha}}_{r}, \overline{\boldsymbol{\beta}}_{r}\right)$ is equal to

$$
r=\frac{\bar{\alpha}_{i j}}{\bar{\beta}_{i j}}, \quad\left\{\begin{array}{l}
i=3,4, \ldots, s+1 \\
j=2,3, \ldots, i-1
\end{array}\right.
$$

and satisfies conditions (71),

under the assumption that all coefficients of (36) are nonnegative, with the convention that ratios $a / 0=+\infty$, and ratios $0 / 0$ are ignored.

\section{Contractivity preserving (CP) Form of $y_{n+1}^{\prime}$ of $\operatorname{CPRKN}(s, p)$ For DERIVING CP PROPERTY}

Similar to the Shu-Osher form of RK methods [12], equation (15) of $y_{n+1}^{\prime}$ can be written in the following $\mathrm{CP}$ form,

$$
y_{n+1}^{\prime}=\alpha_{s+1,1} y_{n}^{\prime}+\Delta t \beta_{s+1,1} y_{n}^{\prime \prime}+\sum_{j=2}^{s}\left[\alpha_{s+1, j} Y_{j}^{\mathrm{sub}}+\Delta t \beta_{s+1, j} Y_{j}^{\prime \prime}\right],
$$

with consistency condition:

$$
\alpha_{s+1,1}+\sum_{j=2}^{s} \alpha_{s+1, j} c_{j}=1
$$

To find the relation between the CP form and the usual form coefficients, comparing (73) with (15), we have the following relations between the CP form coefficients and the usual form coefficients (here $Y_{j}^{\text {sub }}, j=2,3, \ldots, s$ in (73) are replaced by the formula (36) for $j=2,3, \ldots, s$, respectively), 


$$
\left.\begin{array}{l}
\left.\beta_{s+1, j}=b_{j}-\left(\sum_{k=j+1}^{s} \alpha_{s+1, k} \bar{a}_{k j}\right)\right\} j=s, s-1, \ldots, 2, \\
\alpha_{s+1, j}=r \beta_{s+1, j} \\
\beta_{s+1,1}=b_{1}-\left(\sum_{k=2}^{s} \alpha_{s+1, k} \bar{a}_{k 1}\right) \\
\alpha_{s+1,1}=1-\left(\sum_{k=2}^{s} \alpha_{s+1, k} c_{k}\right)
\end{array}\right\} j=1 .
$$

Using the order mentioned above of substitution operations, relations (75) and (76) allow a simple transformation of the usual coefficients of (15) to convenient CP coefficients of (73) for deriving CP property.

It is to be noted that one can also obtain relations (75) and (76) by using naturally the canonical CP form (67) and the form (68) with $\bar{b}_{i}$ (in $\overline{\boldsymbol{\beta}}_{0}$ and $\overline{\boldsymbol{w}}_{0}$ ) replaced by $b_{i}, i=1,2, \ldots, s$.

Form (73) is called the CP form of $y_{n+1}^{\prime}$. We can rearrange $y_{n+1}^{\prime}$ in (73) as follows:

$$
y_{n+1}^{\prime}=\alpha_{s+1,1}\left[y_{n}^{\prime}+\Delta t \frac{\beta_{s+1,1}}{\alpha_{s+1,1}} y_{n}^{\prime \prime}\right]+\sum_{j=2}^{s} \alpha_{s+1, j}\left[Y_{j}^{\text {sub }}+\Delta t \frac{\beta_{s+1, j}}{\alpha_{s+1, j}} Y_{j}^{\prime \prime}\right],
$$

with consistency condition (74).

Thus we obtain the difference $y_{n+1}^{\prime}-\tilde{y}_{n+1}^{\prime}$ from (77) as follows:

$$
\begin{aligned}
& y_{n+1}^{\prime}-\tilde{y}_{n+1}^{\prime}=\alpha_{s+1,1}\left[\left(y_{n}^{\prime}+\Delta t \frac{\beta_{s+1,1}}{\alpha_{s+1,1}} y_{n}^{\prime \prime}\right)-\left(\tilde{y}_{n}^{\prime}+\Delta t \frac{\beta_{s+1,1}}{\alpha_{s+1,1}} \tilde{y}_{n}^{\prime \prime}\right)\right] \\
& \quad+\sum_{j=2}^{s} \alpha_{s+1, j}\left[\left(Y_{j}^{\text {sub }}+\Delta t \frac{\beta_{s+1, j}}{\alpha_{s+1, j}} Y_{j}^{\prime \prime}\right)-\left(\tilde{Y}_{j}^{\text {sub }}+\Delta t \frac{\beta_{s+1, j}}{\alpha_{s+1, j}} \tilde{Y}_{j}^{\prime \prime}\right)\right] .
\end{aligned}
$$

To find the CP coefficient of $y_{n+1}^{\prime}$ of $\operatorname{CPRKN}(s, p)$, it is natural and useful to consider a particular CP form (77) where $\left(\alpha_{s+1, j}, \beta_{s+1, j}\right), j=2,3, \ldots, s$ satisfy the same ratio $r$ defined in (61) for $\boldsymbol{Y}^{\text {sub }}$,

$$
r=\frac{\alpha_{s+1, j}}{\beta_{s+1, j}}, \quad j=2,3, \ldots, s \quad \text { and } \quad r \leq \frac{\alpha_{s+1,1}}{\beta_{s+1,1}} .
$$

Provided all the coefficients of (77) are nonnegative, the following straightforward extension of a result presented in $[2,5]$ holds. 
T. Nguyen-Ba, T. Giordano, R. Vaillancourt - On RKN formulae ...

Theorem 3. If $f$ satisfies conditions (7), (8) and (9) of the FE method, then $y_{n+1}^{\prime}$ of $(77)$ of $\operatorname{CPRKN}(s, p)$ method satisfies the CP property

$$
\left\|y_{n+1}^{\prime}-\tilde{y}_{n+1}^{\prime}\right\| \leq\left\|y_{n}^{\prime}-\tilde{y}_{n}^{\prime}\right\|
$$

provided

$$
\Delta t \leq c_{\text {feasible }}^{\prime} \Delta t_{\mathrm{FE}}
$$

where the feasible CP coefficient $c_{\text {feasible }}^{\prime}$ is equal to $r$ of (79) under the assumption that all coefficients of (77) are nonnegative, with the convention that the ratios $a / 0=+\infty$, and the ratios $0 / 0$ are ignored.

Proof. The proof of this theorem is similar to the proof of theorem 1.

It is to be noted here that each representation (77) of $y_{n+1}^{\prime}$ (to obtain $y_{n+1}^{\prime}$ to order $p$ ) with coefficients $\alpha_{s+1, j}, \beta_{s+1, j}$ will produce a feasible $\mathrm{CP}$ coefficient, $c_{\text {feasible }}^{\prime}$, defined in Theorem 3 and a feasible $y_{n+1}^{\prime}$ of $\operatorname{CPRKN}(s, p)$ in CP form (77). What we really want is not merely a feasible $y_{n+1}^{\prime}$ of method $\operatorname{CPRKN}(s, p)$ in $\mathrm{CP}$ form but a best $y_{n+1}^{\prime}$. This question will be considered in Section 6 .

\section{Optimizing CP Coefficient $c(\operatorname{CPRKN}(s, p))$ of $\operatorname{CPRKN}(s, p)$}

We can now formulate the problem to optimize simultaneously

- formulae for $Y_{i}^{\mathrm{sub}}, i=2,3, \ldots, s+1$ using the CP form (68) which is written solely in terms of the vectors and matrices of the usual form,

- formula for $y_{n+1}^{\prime}$, using the usual form (15) and the CP form (77),

and obtain the $\mathrm{CP}$ coefficient $c(\operatorname{CPRKN}(s, p))$.

Hence, the problem of optimizing $\operatorname{CPRKN}(s, p)$ can be formulated as

maximize $r$,

subject to

- the componentwise inequalities for $Y_{i}^{\mathrm{sub}}$,

$$
\begin{aligned}
\overline{\boldsymbol{v}}_{r} & =\left(\boldsymbol{I}+r \overline{\boldsymbol{\beta}}_{0}\right)^{-1} \overline{\boldsymbol{v}}_{0} \geq 0, \\
\overline{\boldsymbol{w}}_{r} & =\left(\boldsymbol{I}+r \overline{\boldsymbol{\beta}}_{0}\right)^{-1} \overline{\boldsymbol{w}}_{0} \geq 0, \\
\overline{\boldsymbol{\beta}}_{r} & =\overline{\boldsymbol{\beta}}_{0}\left(\boldsymbol{I}+r \overline{\boldsymbol{\beta}}_{0}\right)^{-1} \geq 0,
\end{aligned}
$$

together with conditions (71) and order conditions mentioned in (32) for $\operatorname{RKN}(s, p)$ to obtain $Y_{s+1}=y_{n+1}$ to order $p$, 
- the following inequalities for $y_{n+1}^{\prime}$,

$$
\begin{aligned}
& b_{i} \geq 0, i=1,2, \ldots, s, \\
& \alpha_{s+1, j} \geq 0, j=1,2, \ldots, s, \\
& \beta_{s+1, j} \geq 0, \quad j=1,2, \ldots, s,
\end{aligned}
$$

together with conditions (79) and order conditions mentioned in (32) for $\operatorname{RKN}(s, p)$ to obtain $y_{n+1}^{\prime}$ to order $p$.

Here a maximized $r$ is the $\mathrm{CP}$ coefficient $c(\operatorname{CPRKN}(s, p))$.

Since the consistency condition (70) is satisfied, condition (81) is equivalent to the following condition, in vector notation,

$$
r \overline{\boldsymbol{\beta}}_{0}\left(\boldsymbol{I}+r \overline{\boldsymbol{\beta}}_{0}\right)^{-1} \boldsymbol{e}_{s+1} \leq \boldsymbol{e}_{s+1} .
$$

It is to be noted here that

- each representation of the canonical CP form (67) of $\boldsymbol{Y}^{\text {sub }}$ with coefficients $\left(\overline{\boldsymbol{v}}_{r}, \overline{\boldsymbol{w}}_{r}, \overline{\boldsymbol{\alpha}}_{r}, \overline{\boldsymbol{\beta}}_{r}\right)$, which satisfy conditions (81)-(83) together with conditions (71) and order conditions mentioned in $(32)$ for $\operatorname{RKN}(s, p)$ to obtain $y_{n+1}$ to order $p$, will produce a feasible CP coefficient

$r=c\left(\overline{\boldsymbol{v}}_{r}, \overline{\boldsymbol{w}}_{r}, \overline{\boldsymbol{\alpha}}_{r}, \overline{\boldsymbol{\beta}}_{r}\right)$ and a feasible $Y_{i}^{\mathrm{sub}}, i=2,3, \ldots, s+1$, in CP form (36),

- each representation of the $\mathrm{CP}$ form (77) of $y_{n+1}^{\prime}$ with coefficients $b_{i}, \alpha_{s+1, j}, \beta_{s+1, j}$ which satisfy conditions (84)-(86) together with conditions (79) and order conditions mentioned in (32) for $\operatorname{RKN}(s, p)$ to obtain $y_{n+1}^{\prime}$ to order $p$, will produce a feasible $\mathrm{CP}$ coefficient $r$ and a feasible $y_{n+1}^{\prime}$, in CP form (77).

\section{Intervals of Absolute stability of $\operatorname{CPRKN}(4,4)$ And $\operatorname{CPRKN}(6,6)$}

To obtain the numerical stability of the new methods, we consider the linear test equation

$$
y^{\prime}=\lambda y, \quad y_{0}=1,
$$

similar to Huang and Innanen [6].

For given numbers $s$ and $p$ of $\operatorname{CPRKN}(s, p)$, we have the following equations:

1. the difference equation and corresponding characteristic equation

$$
\eta_{0}(z) y_{n}+\eta_{1}(z) y_{n+1}=0, \quad \eta_{0}(z) y_{n}+\eta_{1}(z) r=0,
$$

(for $y$ of $\operatorname{CPRKN}(s, p)$ ) obtained by applying the predictors $\mathrm{P}_{2}, \mathrm{P}_{3}, \ldots, \mathrm{P}_{s}$ (13) and the integration formula IF (14) with constant step size $h$ to the test equation (87). Here $z=\lambda h$. 
T. Nguyen-Ba, T. Giordano, R. Vaillancourt - On RKN formulae ...

Table 1: Intervals of absolute stability for $y$ and $y^{\prime}$ of $\operatorname{CPRKN}(4,4)$ and of $\operatorname{CPRKN}(6,6)$ respectively.

\begin{tabular}{|l|l|l|}
\hline & \multicolumn{2}{|l|}{ Interval of absolute stability for } \\
Method & $y$ & $y^{\prime}$ \\
\hline CPRKN $(4,4)$ & $(-3.92,0)$ & $(-4.00,0)$ \\
CPRKN $(6,6)$ & $(-4.96,0)$ & $(-4.96,0)$ \\
\hline
\end{tabular}

2. the difference equation and corresponding characteristic equation

$$
\eta_{0}^{\prime}(z) y_{n}+\eta_{1}^{\prime}(z) y_{n+1}=0, \quad \eta_{0}^{\prime}(z) y_{n}+\eta_{1}^{\prime}(z) r=0,
$$

(for $y^{\prime}$ ) obtained by applying the predictors $\mathrm{P}_{2}, \mathrm{P}_{3}, \ldots, \mathrm{P}_{s}$ and the integration formula IF' (15).

Similar to Dormand et al. [1], we have two regions of absolute stability $\mathcal{R}$ and $\mathcal{R}^{\prime}$ : a complex number $z$ is in $\mathcal{R}$ for $y$ and $\mathcal{R}^{\prime}$ for $y^{\prime}$ if the root of the characteristic equation in (88) and (89), respectively, satisfies the root condition (see [9, pp. 70]).

The scanning method used to find the regions of absolute stability is similar to the one used for Runge-Kutta methods (see [9]).

The intervals of absolute stability for $y$ and $y^{\prime}$ of $\operatorname{CPRKN}(4,4)$ and of $\operatorname{CPRKN}(6,6)$, respectively, are shown in Table 1.

\section{Numerical RESUlts}

The relative energy error $(\operatorname{EE}(t))$ at time $t$ is defined as

$$
\mathrm{EE}(t)=\left|\frac{\mathrm{E}(t)-\mathrm{E}(0)}{\mathrm{E}(0)}\right|
$$

where $\mathrm{E}(t)$ is the energy at time $t$.

We compare the relative energy error $(\mathrm{EE}(t))$ as a function of number of function evaluations (NFE) of CPRKN $(4,4), \operatorname{CPRKN}(6,6)$, Dormand-El-Mikkawy-Prince $\operatorname{DEP}(4,3) 4 \mathrm{FM}$ and $\operatorname{DEP}(6,4) 6 \mathrm{FM}[1]$ after a 1000 periods integration of a Hamiltonian system (Huang and Innanen [6]) using fixed step sizes.

Tables 2, 3 and 4 list relative energy errors (EE) at $t=2000 \pi$ as a function of number of function evaluations (NFE) for $\operatorname{CPRKN}(4,4), \operatorname{CPRKN}(6,6), \operatorname{DEP}(4,3) 4 \mathrm{FM}$ and $\mathrm{DEP}(6,4) 6 \mathrm{FM}$ after a 1000 periods integration of Kepler's two-body problem with eccentricities $e=0.3,0.5,0.7$ respectively. It is seen, from Tables 2,3 and 
T. Nguyen-Ba, T. Giordano, R. Vaillancourt - On RKN formulae ...

Table 2: Relative energy error (EE) at $t=2000 \pi$ as a function of number of function evaluations (NFE) after a 1000 periods integration of Kepler's two-body problem with eccentricity $e=0.3$.

\begin{tabular}{|c|c|c||c|c|c|}
\hline & \multicolumn{2}{|c||}{ Relative energy error in } & \multicolumn{2}{c|}{ Relative energy error in } \\
NFE & CPRKN $(4,4)$ & DEP $(4,3) 4$ FM & NFE & CPRKN $(6,6)$ & DEP $(6,4) 6$ FM \\
\hline $2.24 \mathrm{e}+05$ & $3.55 \mathrm{e}-04$ & $2.82 \mathrm{e}-03$ & $1.50 \mathrm{e}+05$ & $4.48 \mathrm{e}-04$ & $5.75 \mathrm{e}-04$ \\
$7.44 \mathrm{e}+05$ & $8.99 \mathrm{e}-07$ & $7.00 \mathrm{e}-06$ & $2.64 \mathrm{e}+05$ & $9.30 \mathrm{e}-06$ & $1.11 \mathrm{e}-05$ \\
$1.26 \mathrm{e}+06$ & $6.36 \mathrm{e}-08$ & $4.95 \mathrm{e}-07$ & $3.78 \mathrm{e}+05$ & $7.70 \mathrm{e}-07$ & $9.04 \mathrm{e}-07$ \\
$1.78 \mathrm{e}+06$ & $1.14 \mathrm{e}-08$ & $8.85 \mathrm{e}-08$ & $4.92 \mathrm{e}+05$ & $1.23 \mathrm{e}-07$ & $1.43 \mathrm{e}-07$ \\
$2.30 \mathrm{e}+06$ & $3.16 \mathrm{e}-09$ & $2.47 \mathrm{e}-08$ & $6.06 \mathrm{e}+05$ & $2.86 \mathrm{e}-08$ & $3.35 \mathrm{e}-08$ \\
$2.82 \mathrm{e}+06$ & $1.14 \mathrm{e}-09$ & $8.92 \mathrm{e}-09$ & $7.20 \mathrm{e}+05$ & $8.59 \mathrm{e}-09$ & $1.00 \mathrm{e}-08$ \\
$3.34 \mathrm{e}+06$ & $4.91 \mathrm{e}-10$ & $3.83 \mathrm{e}-09$ & $8.34 \mathrm{e}+05$ & $3.07 \mathrm{e}-09$ & $3.60 \mathrm{e}-09$ \\
$3.86 \mathrm{e}+06$ & $2.38 \mathrm{e}-10$ & $1.86 \mathrm{e}-09$ & $9.48 \mathrm{e}+05$ & $1.25 \mathrm{e}-09$ & $1.47 \mathrm{e}-09$ \\
$4.38 \mathrm{e}+06$ & $1.27 \mathrm{e}-10$ & $9.90 \mathrm{e}-10$ & $1.06 \mathrm{e}+06$ & $5.67 \mathrm{e}-10$ & $6.65 \mathrm{e}-10$ \\
$4.90 \mathrm{e}+06$ & $7.22 \mathrm{e}-11$ & $5.65 \mathrm{e}-10$ & $1.18 \mathrm{e}+06$ & $2.78 \mathrm{e}-10$ & $3.26 \mathrm{e}-10$ \\
$5.42 \mathrm{e}+06$ & $4.38 \mathrm{e}-11$ & $3.42 \mathrm{e}-10$ & $1.29 \mathrm{e}+06$ & $1.45 \mathrm{e}-10$ & $1.71 \mathrm{e}-10$ \\
\hline
\end{tabular}

4, that $\operatorname{CPRKN}(4,4)$ and $\operatorname{CPRKN}(6,6)$ compare favorably with $\operatorname{DEP}(4,3) 4 \mathrm{FM}$ and $\operatorname{DEP}(6,4) 6 \mathrm{FM}$.

The NFE percentage efficiency gain (NFE PEG) is defined by the formula (cf. Sharp [11]),

$$
(\mathrm{NFE} \mathrm{PEG})=100 \sum_{j}\left[\frac{\mathrm{NFE}_{1, j}}{\mathrm{NFE}_{2, j}}-1\right],
$$

where $\mathrm{NFE}_{1, j}$ and $\mathrm{NFE}_{2, j}$ are the $\mathrm{NFE}$ of methods 1 and 2 , respectively, associated with a given problem $\mathrm{P}$, and $j=-\log _{10}(\mathrm{EE})$. The NFE was obtained from the curves which fit, in a least-squares sense, the data $\left(\log _{10}(\mathrm{EE}), \log _{10}(\mathrm{NFE})\right)$ by means of MATLAB's polyfit.

Table 5 lists the NFE PEGs of CPRKN $(4,4)$ and $\operatorname{CPRKN}(6,6)$ over $\operatorname{DEP}(4,3) 4 \mathrm{FM}$ and $\operatorname{DEP}(6,4) 6 \mathrm{FM}$ after a 1000 periods integration of Kepler's two-body problem with $e=0.3,0.5,0.7$ respectively. It is seen that $\operatorname{CPRKN}(4,4)$ wins over $\operatorname{DEP}(4,3) 4 \mathrm{FM}$ and $\operatorname{CPRKN}(6,6)$ is similar to $\operatorname{DEP}(6,4) 6 \mathrm{FM}$. 
T. Nguyen-Ba, T. Giordano, R. Vaillancourt - On RKN formulae ...

Table 3: Relative energy error (EE) at $t=2000 \pi$ as a function of number of function evaluations (NFE) after a 1000 periods integration of Kepler's two-body problem with eccentricity $e=0.5$.

\begin{tabular}{|c|c|c||c|c|c|}
\hline & \multicolumn{2}{|c||}{ Relative energy error in } & \multicolumn{2}{c|}{ Relative energy error in } \\
NFE & CPRKN $(4,4)$ & DEP $(4,3) 4$ FM & NFE & CPRKN $(6,6)$ & DEP $(6,4) 6$ FM \\
\hline $7.20 \mathrm{e}+05$ & $2.65 \mathrm{e}-05$ & $2.03 \mathrm{e}-04$ & $4.80 \mathrm{e}+05$ & $1.34 \mathrm{e}-05$ & $1.70 \mathrm{e}-05$ \\
$1.55 \mathrm{e}+06$ & $5.81 \mathrm{e}-07$ & $4.43 \mathrm{e}-06$ & $7.20 \mathrm{e}+05$ & $8.05 \mathrm{e}-07$ & $9.98 \mathrm{e}-07$ \\
$2.38 \mathrm{e}+06$ & $6.84 \mathrm{e}-08$ & $5.21 \mathrm{e}-07$ & $9.60 \mathrm{e}+05$ & $1.08 \mathrm{e}-07$ & $1.33 \mathrm{e}-07$ \\
$3.20 \mathrm{e}+06$ & $1.53 \mathrm{e}-08$ & $1.17 \mathrm{e}-07$ & $1.20 \mathrm{e}+06$ & $2.28 \mathrm{e}-08$ & $2.80 \mathrm{e}-08$ \\
$4.03 \mathrm{e}+06$ & $4.86 \mathrm{e}-09$ & $3.70 \mathrm{e}-08$ & $1.44 \mathrm{e}+06$ & $6.38 \mathrm{e}-09$ & $7.83 \mathrm{e}-09$ \\
$4.86 \mathrm{e}+06$ & $1.91 \mathrm{e}-09$ & $1.46 \mathrm{e}-08$ & $1.68 \mathrm{e}+06$ & $2.17 \mathrm{e}-09$ & $2.67 \mathrm{e}-09$ \\
$5.69 \mathrm{e}+06$ & $8.70 \mathrm{e}-10$ & $6.63 \mathrm{e}-09$ & $1.92 \mathrm{e}+06$ & $8.54 \mathrm{e}-10$ & $1.05 \mathrm{e}-09$ \\
$6.52 \mathrm{e}+06$ & $4.41 \mathrm{e}-10$ & $3.36 \mathrm{e}-09$ & $2.16 \mathrm{e}+06$ & $3.75 \mathrm{e}-10$ & $4.60 \mathrm{e}-10$ \\
$7.34 \mathrm{e}+06$ & $2.43 \mathrm{e}-10$ & $1.85 \mathrm{e}-09$ & $2.40 \mathrm{e}+06$ & $1.79 \mathrm{e}-10$ & $2.20 \mathrm{e}-10$ \\
$8.17 \mathrm{e}+06$ & $1.42 \mathrm{e}-10$ & $1.08 \mathrm{e}-09$ & $2.64 \mathrm{e}+06$ & $9.20 \mathrm{e}-11$ & $1.13 \mathrm{e}-10$ \\
$9.00 \mathrm{e}+06$ & $8.79 \mathrm{e}-11$ & $6.69 \mathrm{e}-10$ & $2.88 \mathrm{e}+06$ & $5.02 \mathrm{e}-11$ & $6.15 \mathrm{e}-11$ \\
\hline
\end{tabular}

Table 4: Relative energy error (EE) at $t=2000 \pi$ as a function of number of function evaluations (NFE) after a 1000 periods integration of Kepler's two-body problem with eccentricity $e=0.7$.

\begin{tabular}{|c|c|c||c|c|c|}
\hline & \multicolumn{2}{|c||}{ Relative energy error in } & \multicolumn{2}{c|}{ Relative energy error in } \\
NFE & CPRKN $(4,4)$ & DEP $(4,3) 4$ FM & NFE & CPRKN $(6,6)$ & DEP $(6,4) 6$ FM \\
\hline $1.04 \mathrm{e}+06$ & $4.23 \mathrm{e}-04$ & $3.29 \mathrm{e}-03$ & $1.08 \mathrm{e}+06$ & $2.77 \mathrm{e}-05$ & $3.59 \mathrm{e}-05$ \\
$2.74 \mathrm{e}+06$ & $3.45 \mathrm{e}-06$ & $2.61 \mathrm{e}-05$ & $1.50 \mathrm{e}+06$ & $2.85 \mathrm{e}-06$ & $3.61 \mathrm{e}-06$ \\
$4.43 \mathrm{e}+06$ & $3.11 \mathrm{e}-07$ & $2.35 \mathrm{e}-06$ & $1.92 \mathrm{e}+06$ & $5.11 \mathrm{e}-07$ & $6.43 \mathrm{e}-07$ \\
$6.13 \mathrm{e}+06$ & $6.15 \mathrm{e}-08$ & $4.65 \mathrm{e}-07$ & $2.34 \mathrm{e}+06$ & $1.29 \mathrm{e}-07$ & $1.61 \mathrm{e}-07$ \\
$7.82 \mathrm{e}+06$ & $1.81 \mathrm{e}-08$ & $1.37 \mathrm{e}-07$ & $2.76 \mathrm{e}+06$ & $4.06 \mathrm{e}-08$ & $5.09 \mathrm{e}-08$ \\
$9.52 \mathrm{e}+06$ & $6.80 \mathrm{e}-09$ & $5.14 \mathrm{e}-08$ & $3.18 \mathrm{e}+06$ & $1.51 \mathrm{e}-08$ & $1.89 \mathrm{e}-08$ \\
$1.12 \mathrm{e}+07$ & $3.00 \mathrm{e}-09$ & $2.26 \mathrm{e}-08$ & $3.60 \mathrm{e}+06$ & $6.34 \mathrm{e}-09$ & $7.94 \mathrm{e}-09$ \\
$1.29 \mathrm{e}+07$ & $1.48 \mathrm{e}-09$ & $1.12 \mathrm{e}-08$ & $4.02 \mathrm{e}+06$ & $2.93 \mathrm{e}-09$ & $3.67 \mathrm{e}-09$ \\
$1.46 \mathrm{e}+07$ & $8.00 \mathrm{e}-10$ & $6.05 \mathrm{e}-09$ & $4.44 \mathrm{e}+06$ & $1.46 \mathrm{e}-09$ & $1.83 \mathrm{e}-09$ \\
$1.63 \mathrm{e}+07$ & $4.62 \mathrm{e}-10$ & $3.49 \mathrm{e}-09$ & $4.86 \mathrm{e}+06$ & $7.78 \mathrm{e}-10$ & $9.75 \mathrm{e}-10$ \\
$1.80 \mathrm{e}+07$ & $2.81 \mathrm{e}-10$ & $2.13 \mathrm{e}-09$ & $5.28 \mathrm{e}+06$ & $4.35 \mathrm{e}-10$ & $5.46 \mathrm{e}-10$ \\
\hline
\end{tabular}


T. Nguyen-Ba, T. Giordano, R. Vaillancourt - On RKN formulae ...

Table 5: NFE PEG of $\operatorname{CPRKN}(4,4)$ over $\operatorname{DEP}(4,3) 4 \mathrm{FM}$ and $\operatorname{CPRKN}(6,6)$ over $\operatorname{DEP}(6,4) 6 \mathrm{FM}$ after a 1000 periods integration of Kepler's two-body problem with $e=0.3, e=0.5$ and $e=0.7$, respectively.

\begin{tabular}{|l|l|l|l|}
\hline \multirow{2}{*}{ method } & \multicolumn{4}{|c|}{$\begin{array}{c}\text { NFE PEG over DEP(4,3)4FM for } \\
\text { two-body problem with: }\end{array}$} \\
\hline CPRKN $(4,4)$ & $e=0.3$ & $e=0.5$ & $e=0.7$ \\
\hline \hline & $51 \%$ & $50 \%$ & $50 \%$ \\
method & \multicolumn{4}{|c|}{ NFE PEG over DEP(6,4)6FM for } \\
two-body problem with:
\end{tabular}

\section{Conclusion}

We constructed new optimal, explicit, $s$-stage Runge-Kutta-Nystrom method of order $p, p=3,4, \ldots, 6(\mathrm{CPRKN}(s, p))$, that have contractivity-preserving properties and nonnegative coefficients.

On the basis of NFE versus the relative energy error, selected $\operatorname{CPRKN}(4,4)$ and CPRKN $(6,6)$ compare favorably with Dormand-El-Mikkawy-Prince DEP $(4,3) 4 \mathrm{FM}$ and $\mathrm{DEP}(6,4) 6 \mathrm{FM}$, respectively, in solving Kepler's problem with varying eccentricity over an interval of 1000 periods.

In the light of the results obtained in this paper, these new $\operatorname{CPRKN}(s, p)$, $p=3,4, \ldots, 6$ appear to be promising solvers which can be combined with Taylor series or CP HO series methods developed earlier [10] to form new higher order methods with contractivity-preserving $(\mathrm{CP})$ properties and nonnegative coefficients for solving efficiently the second-order system of non-stiff ordinary differential equations.

Acknowledgment This work was supported in part by the Natural Sciences and Engineering Research Council of Canada. 
T. Nguyen-Ba, T. Giordano, R. Vaillancourt - On RKN formulae ...

A. Coefficients of $\operatorname{CPRKN}(s, p), p=3,4, \ldots, 6$

The appendix lists the usual form of five new $\operatorname{CPRKN}(s, p)$ methods with their $\mathrm{CP}$ coefficient $c(\operatorname{CPRKN}(s, p))$.

$\operatorname{CPRKN}(\mathbf{2}, \mathbf{3}) \cdot c(\operatorname{CPRKN}(2,3))=1.5$.

\begin{tabular}{c|cc}
0 & & $\bar{a}_{i j}$ \\
$\frac{2}{3}$ & $\frac{2}{9}$ & \\
\hline $\bar{b}_{i}$ & $\frac{1}{4}$ & $\frac{1}{4}$ \\
\hline$b_{i}$ & $\frac{1}{4}$ & $\frac{3}{4}$
\end{tabular}

CPRKN $(3,4) . c(\operatorname{CPRKN}(3,4))=2.2542293787479135$.

\begin{tabular}{c|ccc}
0 & & & $\bar{a}_{i j}$ \\
$\frac{5703594}{16064153}$ & $\frac{547322}{8683431}$ & & \\
$\frac{10360559}{12261757}$ & $\frac{112823}{2496535}$ & $\frac{4709345}{15104824}$ & \\
\hline $\bar{b}_{i}$ & $\frac{1}{9}$ & $\frac{1885193}{5703594}$ & $\frac{499307}{8555391}$ \\
\hline$b_{i}$ & $\frac{1}{9}$ & $\frac{20603748}{40203547}$ & $\frac{2862467}{7604792}$
\end{tabular}

CPRKN(4,4). $c(\operatorname{CPRKN}(4,4))=2.4743852177875874$.

\begin{tabular}{c|cccc}
0 & & & & \\
$\frac{26971918}{107581049}$ & $\frac{11868682}{377642077}$ & & $\bar{a}_{i j}$ & \\
$\frac{58977037}{101250069}$ & $\frac{972878}{65595991}$ & $\frac{41074969}{265316004}$ & & \\
$\frac{23277231}{26105459}$ & $\frac{83526627}{846839644}$ & $\frac{44674505}{248163904}$ & $\frac{15185060}{127738057}$ & \\
\hline $\bar{b}_{i}$ & $\frac{26994554}{328987169}$ & $\frac{53393375}{207511886}$ & $\frac{208549974}{1569486133}$ & $\frac{25168925}{906469463}$ \\
\hline$b_{i}$ & $\frac{17891713}{218049315}$ & $\frac{14894263}{43373362}$ & $\frac{40778691}{128129371}$ & $\frac{27846884}{108654621}$
\end{tabular}


T. Nguyen-Ba, T. Giordano, R. Vaillancourt - On RKN formulae ...

CPRKN (5,5). $c(\operatorname{CPRKN}(5,5))=2.4307903085928104$.

\begin{tabular}{c|cccccc}
0 & \multicolumn{5}{|c}{} \\
$\frac{68909267}{178744101}$ & $\frac{31624111}{425555783}$ & & & $\bar{a}_{i j}$ & \\
$\frac{13013228}{65692391}$ & $\frac{2299759}{274780277}$ & $\frac{5514383}{490121757}$ & & & \\
$\frac{119047355}{176052511}$ & $\frac{1570365}{104029019}$ & $\frac{20347847}{284778633}$ & $\frac{12591039}{88620110}$ & & \\
$\frac{69512934}{74012023}$ & $\frac{12808156}{182165325}$ & $\frac{4231711}{164606135}$ & $\frac{58976315}{260757231}$ & $\frac{182143463}{1532329653}$ & \\
\hline $\bar{b}_{i}$ & $\frac{14520741}{223581817}$ & $\frac{11229819}{101906302}$ & $\frac{46531259}{226905735}$ & $\frac{31617786}{287289619}$ & $\frac{10588203}{1087932953}$ \\
\hline$b_{i}$ & $\frac{14520741}{223581817}$ & $\frac{16327696}{91046147}$ & $\frac{69883863}{273275923}$ & $\frac{1967557}{57884909}$ & $\frac{15571109}{97257192}$
\end{tabular}

CPRKN (6,6) with $c(\operatorname{CPRKN}(6,6))=2.4672918884438562$.

\begin{tabular}{c|ccccccc}
0 & \multicolumn{7}{|c}{} \\
$\frac{6648706}{39027077}$ & $\frac{3999571}{275613952}$ & & & & & & \\
$\frac{30648937}{79250275}$ & $\frac{1350862}{522581577}$ & $\frac{9232128}{127873411}$ & & & & \\
$\frac{75321914}{105966849}$ & $\frac{20814370}{224800513}$ & $\frac{10697606}{442107819}$ & $\frac{47016859}{346130514}$ & & & & \\
$\frac{6255665}{10780901}$ & $\frac{2905627}{204565870}$ & $\frac{18175723}{134876122}$ & $\frac{3672823}{307407819}$ & $\frac{1030929}{138615316}$ & & \\
$\frac{469000023}{506551154}$ & $\frac{16231130}{578987087}$ & $\frac{3336798}{14855867}$ & $\frac{43589951}{610836173}$ & $\frac{8006719}{151269626}$ & $\frac{8085943}{156460637}$ & \\
\hline $\bar{b}_{i}$ & $\frac{10892061}{206668234}$ & $\frac{252458291}{1241932224}$ & $\frac{14535418}{137797841}$ & $\frac{55242801}{1159422986}$ & $\frac{10863867}{140225018}$ & $\frac{4041093}{301275815}$ \\
\hline$b_{i}$ & $\frac{10892061}{2066668234}$ & $\frac{139166744}{567979543}$ & $\frac{24185509}{140610440}$ & $\frac{40325482}{244756631}$ & $\frac{30769025}{166702063}$ & $\frac{106285627}{587407756}$
\end{tabular}

\section{REFERENCES}

[1] J. R. Dormand, M. E. A. El-Mikkawy, P. J. Prince, Families of Runge-KuttaNystrom formulae, IMA J. Numer. Anal. 7 (1987), 235-250.

[2] S. Gottlieb, C. W. Shu, E. Tadmor, Strong stability preserving high-order time discretization methods, SIAM Rev. 43 (2001), 89-112.

[3] S. Gottlieb, D. I. Ketcheson, C. W. Shu, High order strong stability preserving time discretization, J. Sci. Comput. 38(3) (2009), 251-289. doi: 10.1007/s10915-0089239-z. 
T. Nguyen-Ba, T. Giordano, R. Vaillancourt - On RKN formulae ...

[4] S. Gottlieb, D. I. Ketcheson, C. W. Shu, Strong Stability Preserving RungeKutta and Multistep Time Discretizations, World Scientific, Singapore, 2011.

[5] C. Huang, Strong stability preserving hybrid methods, Appl. Numer. Math. 59 (2009), 891-904.

[6] T. Y. Huang, K. Innanen, A survey of multiderivative multistep integrators, Astron. J. 112(3) (1996), 1254-1262.

[7] T. E. Hull, W. H. Enright, B. M. Fellen, A. E. Sedgwick, Comparing numerical methods for ordinary differential equations, SIAM J. Numer. Anal. 9 (1972), 603-637.

[8] C. A. Kennedy, M. K. Carpenter, R. M. Lewis, Low-storage, explicit RungeKutta schemes for the compressible Navier-Stokes equations, Appl. Numer. Math. 35 (2000), 177-219.

[9] J. D. Lambert, Numerical Methods for Ordinary Differential Systems: The Initial Value Problem, Wiley, London, 1991.

[10] T. Nguyen-Ba, T. Giordano, R. Vaillancourt, Contractivity-preserving explicit multistep Hermite-Obrechkoff series differential equation solvers, Celest. Mech. Dyn. Astr. 1244 (2015), 385-404. doi: 10.1007/s10569-015-9668-6

[11] P. W. Sharp, Numerical comparison of explicit Runge-Kutta pairs of orders four through eight, ACM Trans. Math. Software. 17 (1991), 387-409.

[12] C. W. Shu, S. Osher, Efficient implementation of essentially non-oscillatory shock-capturing schemes. J. Comput. Phys. 77 (1988), 439-471.

Truong Nguyen-Ba

Department of Mathematics and Statistics, University of Ottawa, Ottawa, Ontario, Canada K1N 6N5

email: trnguyen@uottawa.ca

Thierry Giordano Department of Mathematics and Statistics, University of Ottawa,

Ottawa, Ontario, Canada K1N 6N5

email: giordano@uottawa.ca

Rémi Vaillancourt

Department of Mathematics and Statistics, 
T. Nguyen-Ba, T. Giordano, R. Vaillancourt - On RKN formulae ...

University of Ottawa,

Ottawa, Ontario,

Canada K1N 6N5

email:remi@uottawa.ca 